\title{
Endovascular treatment of an iatrogenic superior mesenteric arteriovenous fistula after Nissen fundoplication
}

\author{
S. Keisin Wang, Jie Xie, Raghu L. Motaganahalli \\ Division of Vascular Surgery, Department of Surgery, Indiana University School of Medicine, Indianapolis, IN 46202, USA.
}

Correspondence to: Dr. Raghu L. Motaganahalli, Division of Vascular Surgery, Department of Surgery, Indiana University School of Medicine, 1801 N. Senate Blvd MPC2-3500, Indianapolis, IN 46202, USA. E-mail: rmotagan@iupui.edu; Dr. S. Keisin Wang, Division of Vascular Surgery, Department of Surgery, Indiana University School of Medicine, 1801 N. Senate Blvd MPC2-3500, Indianapolis, IN 46202, USA. E-mail: wangkei@iupui.edu

How to cite this article: Wang SK, Xie J, Motaganahalli RL. Endovascular treatment of an iatrogenic superior mesenteric arteriovenous fistula after Nissen fundoplication. Vesse/ P/us 2018;2:7. http://dx.doi.org/10.20517/2574-1209.2018.15

Received: 27 Mar 2018 First Decision: 24 Apr 2018 Revised: 24 Apr 2018 Accepted: 26 Apr 2018 Published: 27 Apr 2018

Science Editor: Mario F. L. Gaudino Copy Editor: Jun-Yao Li Production Editor: Cai-Hong Wang

\begin{abstract}
We present a case of a superior mesenteric arteriovenous fistula (SMAVF) diagnosed four years after index Nissen fundoplication and examine the associated imaging, clinical course, and surgical treatment followed by a review of the limited, available literature. From a transbrachial approach, a covered stent was successfully deployed in the superior mesenteric artery to exclude the fistula after confirmation of the site of pathology on both digital subtraction angiography and intravascular ultrasound. Follow-up imaging demonstrated continued exclusion of the anomalous fistula with complete resolution of his symptoms at both his postprocedure and 1-year follow-up visits. SMAVFs are usually encountered secondary to previous surgical dissection or trauma and presents with nondescript abdominal pain making early diagnosis difficult; however, they can be successfully treated with minimally-invasive stent exclusion.
\end{abstract}

Keywords: Superior mesenteric artery, iatrogenic, fistula, endovascular, stent, treatment

\section{INTRODUCTION}

A superior mesenteric arteriovenous fistula (SMAVF) is a rare pathology that can lead to gastrointestinal bleeding, portal hypertension, and hepatic dysfunction if allowed to progress ${ }^{[1]}$. Although limited outcomes data is available, endovascular therapy is preferred secondary to a lower postoperative morbidity compared to open surgical repair ${ }^{[2]}$.

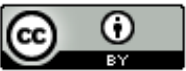

C The Author(s) 2018. Open Access This article is licensed under a Creative Commons Attribution 4.0 International License (https://creativecommons.org/licenses/by/4.0/), which permits unrestricted use, sharing, adaptation, distribution and reproduction in any medium or format, for any purpose, even commercially, as long as you give appropriate credit to the original author(s) and the source, provide a link to the Creative Commons license, and indicate if changes were made.

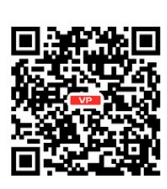




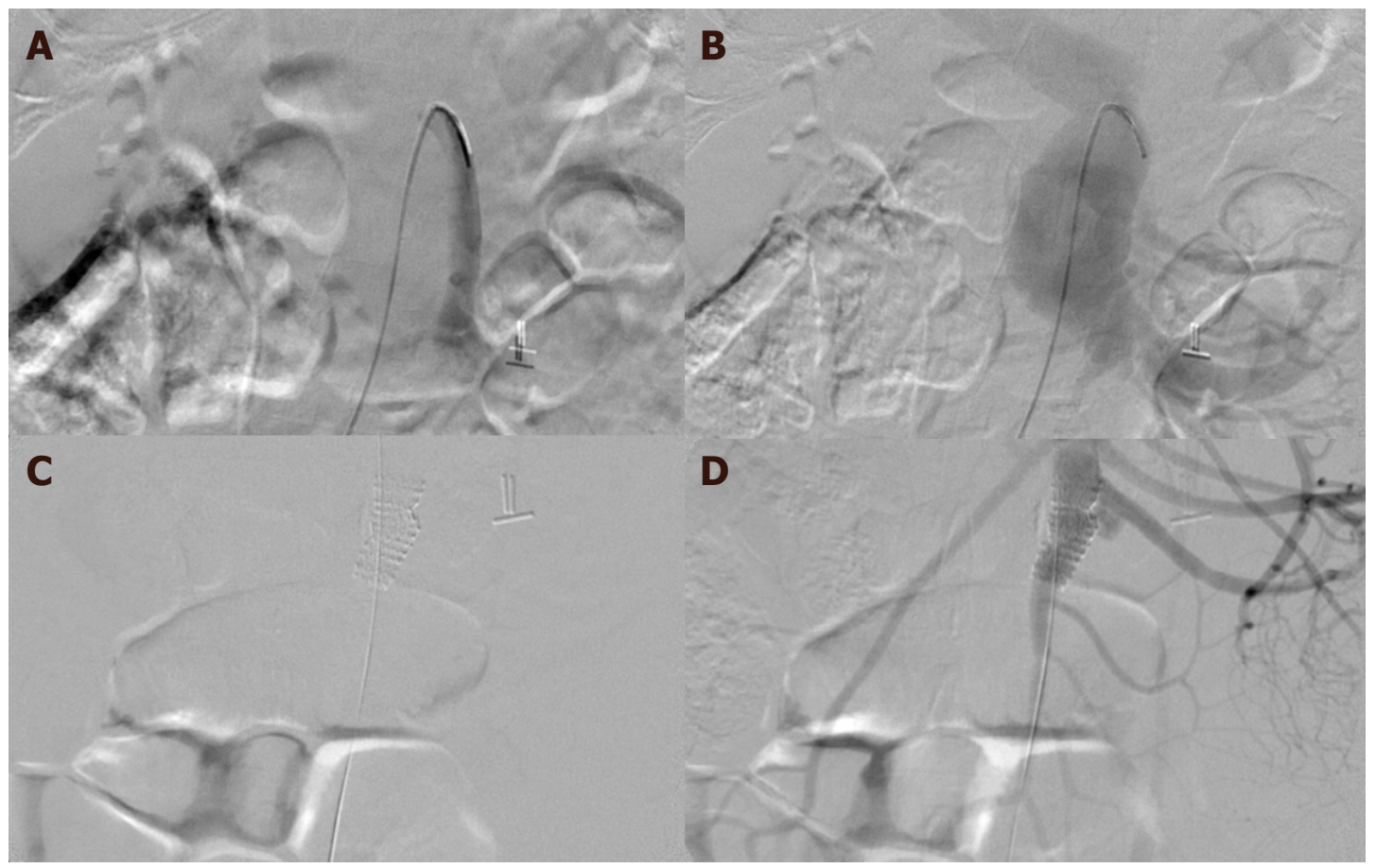

Figure 1. Initial selective angiography demonstrates flow through the proximal SMA (A) before redirection of contrast through the SMV (B) and the venous system. After accessing the brachial artery, a $10 \mathrm{~mm} \times 2.5 \mathrm{~cm}$ Viabahn stent was deployed (C) over the identified SMA defect (via IVUS) successfully excluding the pathologic anatomy (D). SMA: superior mesenteric artery; SMV: superior mesenteric vein; IVUS: intravascular ultrasound

\section{CASE REPORT}

A 44-year-old gentleman presented to us following a 4-year history of nonspecific abdominal discomfort and a newly discovered peri-umbilical bruit. He recalled developing abdominal discomfort immediately following laparoscopic Nissen fundoplication that was complicated by a large peritoneal bleed upon trocar insertion. This bleeding event during the index operation resolved with copious application of surgical clips by the general surgeon. A mesenteric duplex was performed which demonstrated turbulence in the superior mesenteric vein (SMV) distribution. The pathologic connection was confirmed on a computed tomographic angiogram (CTA), but it was difficult to distinguish the exact location of fistula formation.

At this point, we elected to proceed with a diagnostic angiogram via a transfemoral approach. The presence and location of the fistula was established but no intervention was undertaken secondary to the significant angulation of the superior mesenteric artery (SMA). The following week, the patient returned to the operating theatre where a cutdown of the left arm was performed to expose the brachial artery to ease SMA cannulation and stent deployment. The brachial artery was accessed with a micropuncture needle and a long $6 \mathrm{~F}$ sheath was positioned into the distal thoracic aorta with the assistance of an OmniFlush angled catheter (AngioDynamics, Latham, NY). Once the SMA was successfully cannulated, digital subtraction angiography (DSA), once again, confirmed an anomalous connection between the SMA and the hypertrophied SMV; identification of the exact point of fistula formation was achieved with the assistance of intravascular ultrasound (IVUS). The previously positioned $6 \mathrm{~F}$ sheath was upsized to a long $12 \mathrm{~F}$ sheath in preparation of stent deployment. After exchanging the wire for a stiff Amplatz (Cook Medical, Bloomington, IN), a $10 \mathrm{~mm} \times 2.5 \mathrm{~cm}$ Viabahn self-expanding, covered stent (W.L. Gore and Associates, Flagstaff, AZ) was deployed to cover the marked anomalous arteriovenous connection [Figure 1].

The patient tolerated the procedure well without any postoperative complications and was discharged home the following day. His repeat CTA at 1-month postoperatively exhibited a patent stent, absence of any 


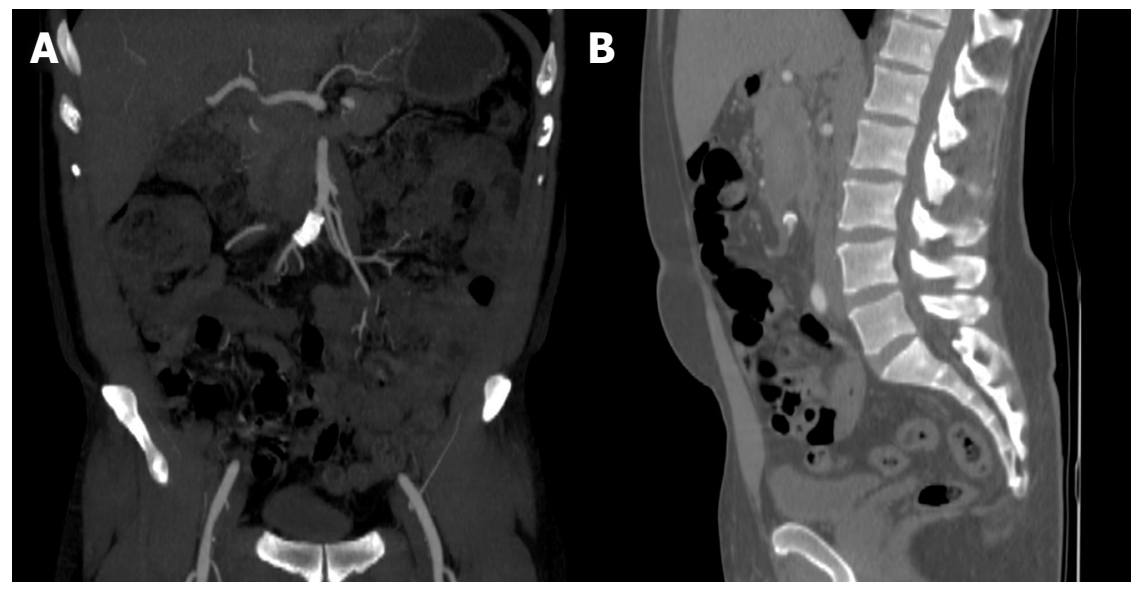

Figure 2. Postoperative surveillance CTA demonstrated successful exclusion of the SMAVF and loss of venous collaterals on both coronal (A) and sagittal (B) projections. CTA: computed tomographic angiogram; SMAVF: superior mesenteric arteriovenous fistula

communicating fistulas, and reduced SMV and portal vein size [Figure 2]. Most importantly, his abdominal discomfort resolved. The patient was again seen in clinic at 14-month postoperatively where a mesenteric duplex demonstrated a widely patent SMA stent in an asymptomatic patient.

\section{DISCUSSION}

SMAVF is most commonly caused by penetrating abdominal trauma or iatrogenic injury during abdominal surgery. Specifically, this complication has been observed after small bowel resection, colectomy, aortobifemoral bypass, and kidney-pancreas transplantation ${ }^{[3,4]}$. The clinical manifestations of SMAVF may vary from completely asymptomatic to nonspecific abdominal pain, anorexia, nausea, diarrhea, gastrointestinal hemorrhage, signs of portal hypertension, and even congestive heart failure secondary to a persistent high-flow state ${ }^{[5]}$. The most common physical findings are the presence of a machine-like bruit, palpable abdominal mass, or a thrill over the abdomen.

Mesenteric duplex can be used as the initial diagnostic imaging modality as it does not have any associated risks related to radiation or contrast administration. While specificity may be high with this study, sensitivity can be lacking - particularly in the morbidly obese patient. Therefore, CTA with both an arterial and venous phase should also be considered as the confirmatory diagnostic modality. However, the gold standard for diagnosis, continues to be intraoperative DSA which carries the highest sensitivity and specificity. In our patient, DSA was used to define the area of vascular defect intraoperatively. Unfortunately, in the setting of significant venous congestion, near-instant filling of arterial and venous collaterals can make delineation of vascular anatomy difficult, even with the benefit or orthogonal angles. In these situations, IVUS in the suspected vascular territories can help identify the exact site of defect for stent coverage.

Endovascular treatment has supplanted open ligation secondary to low morbidity and a desire to avoid laparotomy in a reoperative abdomen. Coil embolization, vascular plugs, and covered stents have all been deployed with varying success. A literature review demonstrates at least 25 instances of endovascular SMAVF repair consisting of 16 cases with coil embolization, two cases with vascular plugs, six cases with covered stents, and one patient repaired with a combination of both coil embolization and covered stent exclusion ${ }^{[5-7]}$. Coil embolization, while effective at fistula exclusion, is associated with a high risk of postoperative migration and inadvertent thrombotic events and should be avoided if possible. For example, three of the coil embolization procedures were complicated by portal thrombosis. While two were selflimited and resolved after heparinization ${ }^{[8,9]}$, the third patient died from multiorgan failure ${ }^{[1]}$. Additionally, 
there was one reported case of bowel ischemia requiring intestinal resection after coil embolization ${ }^{[10]}$. Not surprisingly, it appears that the risk of coil migration is increased in patients with a short, wide fistula neck.

Although no complications were reported in the seven patients treated with covered stents, the drawbacks associated with SMA stent grafting should not be neglected; these potential complications include the need for large access sheaths, difficulty cannulating the SMA ostia, intraoperative thromboembolism, and risk of subacute or late stent thrombosis which could lead to acute or chronic bowel ischemia. Therefore, the risks and benefits for each patient should be individually characterized before an operative decision is made. We empirically recommend dual antiplatelet therapy in the three-month postimplantation period with subsequent life-long treatment with aspirin and regular duplex surveillance for stenosis/occlusion.

In conclusion, SMAVF is a rare pathology observed after abdominal injury. Although severe morbidity can occur if left untreated, these anomalous connections can be safely intervened upon to relieve symptoms with covered stents.

\section{DECLARATIONS}

\section{Authors' contributions}

Concept and design, writing the manuscript, and critical revision: Wang SK, Xie J, Motaganahalli RL

Data collection: Wang SK

\section{Financial support and sponsorship}

None.

\section{Conflicts of interest}

The authors have no relevant financial conflicts of interest to disclose.

\section{Patient consent}

Written informed consent was obtained previous to the preparation and submission of this manuscript and is available upon request.

\section{Ethics approval}

This report was exempt from institutional review board protocols.

\section{Copyright}

(c) The Author(s) 2018.

\section{REFERENCES}

1. Zhao Y, Li Z, Zhang L, Wei B, Zeng X, Fu P. Portal vein thrombosis secondary to embolization of superior mesenteric arteriovenous fistula. Ann Vasc Surg 2014;28:490.e9-12.

2. Bulut T, Oosterhof-Berktas R, Geelkerken RH, Brusse-Keizer M, Stassen EJ, Kolkman JJ. Long-term results of endovascular treatment of atherosclerotic stenoses or occlusions of the coeliac and superior mesenteric artery in patients with mesenteric ischaemia. Eur $J$ Vasc Endovasc Surg 2017;53:583-90.

3. Khan TF, Ciancio G, Burke GW 3rd, Sfakianakis GN, Miller J. Pseudoaneurysm of the superior mesenteric artery with an arteriovenous fistula after simultaneous kidney-pancreas transplantation. Clin Transplant 1999;13:277-9.

4. Grujic D, Knezevic A, Vojvodic S, Grujic B. Superior mesenteric arteriovenous fistula presenting with massive lethal upper gastrointestinal bleeding 14 years after small bowel resection. Balkan Med J 2015;32:214-7.

5. Weinstein D, Altshuler A, Belinki A, Peer A, Gayer G, Halevy A, Bass A. Superior mesenteric artery to superior mesenteric vein arteriovenous fistula presenting as abdominal pain and gastrointestinal bleeding 3 years after an abdominal gunshot wound: report of a case and review of the literature. J Trauma 2009;66:E13-6.

6. Wang C, Zhu X, Guo GH, Shu X, Wang J, Zhu Y, Li B, Wang Y. Superior mesenteric arteriovenous fistula presenting as gastrointestinal 
bleeding: case report and literature review. Rev Esp Enferm Dig 2016;108:503-7.

7. An T, Zhou S, Song J, Jiang T, Li X, Wang W. Massive gastrointestinal bleeding secondary to superior mesenteric arteriovenous fistula. Am J Gastroenterol 2013;108:1662-5.

8. Mick SL, Bush HL Jr, Barie PS. Superior mesenteric arteriovenous fistula causing massive hematemesis. Surgery 2003;134:102-4.

9. Purow DB, Maltz C. Superior mesenteric arteriovenous fistula: a rare cause of esophageal variceal bleeding. J Clin Gastroenterol 2002;35:284-5.

10. Hussein M, Issa G, Muhsen S, Haydar A. Superior mesenteric arteriovenous fistula embolisation complicated by bowel ischaemia. $B M J$ Case Rep 2013;2013:bcr2013009521. 